\title{
High-Sensitivity Troponin T and Copeptin in Non-ST Acute Coronary Syndromes: Implications for Prognosis and Role of hsTnT and Copeptin in Non-STEACS
}

\author{
Diana Hernández-Romero, ${ }^{1}$ José María García-Salas, ${ }^{2}$ Ángel López-Cuenca, ${ }^{1}$ \\ Patricio Pérez-Berbel, ${ }^{3}$ Carmen Puche, ${ }^{2}$ Teresa Casas, ${ }^{2}$ Esteban Orenes-Piñero, ${ }^{1}$ \\ Sergio Manzano-Fernández, ${ }^{1}$ Mariano Valdés, ${ }^{1}$ and Francisco Marín ${ }^{1}$ \\ ${ }^{1}$ Servicio de Cardiología, Hospital Universitario Virgen de la Arrixaca, 30120 Murcia, Spain \\ ${ }^{2}$ Servicio de Análisis Clínicos, Hospital Universitario Virgen de la Arrixaca, 30120 Murcia, Spain \\ ${ }^{3}$ Servicio de Cardiología, Hospital General Universitario de Alicante, 03010 Alicante, Spain \\ Correspondence should be addressed to Diana Hernández-Romero, dianahr@um.es
}

Received 24 October 2011; Accepted 28 November 2011

Academic Editor: Gabriele Di Giammarco

Copyright (c) 2012 Diana Hernández-Romero et al. This is an open access article distributed under the Creative Commons Attribution License, which permits unrestricted use, distribution, and reproduction in any medium, provided the original work is properly cited.

\begin{abstract}
High-sensitivity TnT (hsTnT) has been proposed to improve the diagnosis and stratification in acute coronary syndromes. Copeptin has been proposed for a rapid and accurate rule out of acute myocardial infarction, but some doubts exist about its use out of the first hours from admission. Abnormalities of serum hsTnT and copeptin levels in non-STEACS and negative TnT, could have prognostic implications. Methods. We included 122 non-STEACS patients without raised TnT, 33 disease controls and 43 healthy controls. We measured hsTnT and copeptin levels. Clinical follow-up at 12 months was performed for adverse endpoints. Results. Non-STEACS patients had raised hsTnT compared with both control groups $(P=0.036$ and $P<0.001)$. Copeptin levels were higher in non-STEACS patients than healthy controls $(P=0.021)$, without differences with disease controls. Raised levels of hs-TnT presented prognostic implications [HR 3.29 (95\%CI: 1.33-7.49), $P=0.010$ ]. hs-TnT could be used for invasive approach decision, as it shows prognostic relevance in conservative approach-patients whereas remains unrelevant for catheterized-patients. Copeptin levels were not associated with adverse events. Conclusion. hsTnT levels increased in non-STEACS, were predictive of adverse events and could be important for recommending an invasive management. We cannot confirm a predictive role of copeptin out of the first hours from admission.
\end{abstract}

\section{Introduction}

Acute coronary syndromes (ACSs) present a complex and heterogeneous pathophysiology [1-3] with high morbidity and mortality mainly due to new cardiac ischaemic events [4]. Current stratification of the risk in patients presenting with ACS without ST-segment elevation (non-STEACS) is based on the identification of those patients with higher risk of suffering adverse events (death, recurrent MI or urgent revascularization), estimated in a $15-30 \%$ of non-STEACS patients. However, up to $10 \%$ of patients who classified as low risk show a cardiovascular event at 3 months followup [5]. Regarding this, the current stratification needs to be improved. It has been proposed to identify new biomarkers or even to use a multimarker approach [6].

Cardiac troponins are components of the contractile apparatus of cardiomyocytes and are released during myocardial necrosis in patients with ACS [7]. Serum troponin (Tn) elevation is a specific and well-established necrosis biomarker in ACS, being the only biomarker currently used for risk stratification and guided invasive management decision in non-STEACS $[8,9]$. In patients showing negative Tn elevation, stratification is more complicated and the elec-tive treatment is usually under the criterium of the car-diologist, although there are established recommendations $[10,11]$. Conventional determination methods fail to detect slightly 
increases of Tn, due in part to the delayed and progressive release of the biomarker after the event. It has been shown that even very small elevation in the troponin concentration is associated with increased risk of adverse outcomes in patients with ACS [12]. A recent publication has shown that cardiac troponin $\mathrm{T}$ ( $\mathrm{TnT}$ ) concentrations measured with a highly sensitive assay were significantly associated with the incidence of cardiovascular death and heart failure in stable coronary artery disease after adjustment for other independent prognostic indicators [13]. In the same way, Reichlin et al. found that the diagnostic performance of sensitivity cardiac Tn assays is excellent within the context of the myocardial infarction, and these assays can substantially improve the early diagnosis of acute myocardial infarction, particularly in patients with a recent onset of chest pain [14]. However, the criteria defining a high sensitive TnT (hsTnT) assay are still under debate, whereas a cutoff point of TnT for risk stratification in patients with ACS remains difficult to establish due to the heterogeneity of the used detection techniques among different laboratories [15].

As the released of the necrosis markers after cell damage might explain the weakness in diagnostic performance of conventional Tn assays early after chest pain onset, markers with different pathophysiologic background independent of cell necrosis might help in the diagnosis of cardiovascular diseases [16]. Arginine vasopressin (AVP), also known as the antidiuretic hormone, is a peptide secreted neurohypophyseal hormone and controls osmotic homeostasis [17]. However, vasopressin is difficult to measure because it is unstable and rapidly cleared [18]. Copeptin, a 39-amino acid glycopeptides that comprises the C-terminal part of the AVP precursor, was found to be a stable and sensitive surrogate marker of AVP release. Copeptin is secreted in equimolar amounts, and it can be quickly and reliably measured in unprocessed plasma or serum. The predictive value of this marker has been shown in coronary artery disease [19], and it has been proposed to be of great value in the rapid rule out of acute myocardial infarction used in combination with TnT increased levels [20].

We hypothesized that the use of a marker of cardiac necrosis determined by high sensitivity methods, such as hsTnT, or a pathophysiologically different biomarker reflecting acute endogenous stress, such as copeptin, together with the association to the classical clinical and electrocardiographic parameters, might help to the better stratification and managements as well as in the prognosis of patients with non-STEACS.

\section{Methods}

2.1. Patients Admission and Selection. We prospectively recruited consecutive patients admitted with a final diagnosis of non-STEACS in two tertiary hospitals. Blood samples were collected for all patients within 48 hours of hospital admission, and baseline clinical characteristics were prospectively recorded. Serum TnT was measured at admission using a commercially available third-generation immunoassay (Elecsys troponin T STAT; Roche Diagnostics,
Mannheim, Germany). According to the manufacturers, the lower limit of detection and the concentration with $\leq 10 \%$ precision were 0.01 and $0.035 \mathrm{ng} / \mathrm{mL}$ for $\mathrm{TnT}$. We adopted the recommendations of the Joint Committee of the American College of Cardiology and the European Society of Cardiology [21] to establish the upper limit of normal cutoff value of $0.035 \mathrm{ng} / \mathrm{mL}$ for TnT. All patients included were stratified as intermediate or high risk by 2002 ACC/AHA Guidelines for Diagnostic and Treatment Strategies in the Emergency Department for Patients with ACS [22]. Patients with elevated conventional TnT (TnT $>0.035 \mathrm{mg} / \mathrm{mL}$ ), concomitant neoplastic, infectious, connective tissue, or inflammatory diseases were excluded $(n=74)$.

During the entire hospitalization period, all patients received standard management as recommended for ACS $[10,23]$ and clinical management decisions about each patient were decided by the cardiologist responsible, who was unaware of the patient's hsTnT and copeptin concentrations.

Patients with non-STEACS were compared with a group of subjects with stable coronary artery disease (CAD), defined as patients with previous ( $>6$ months) ST elevation myocardial infarct, non-ST elevation myocardial infarct or stable angina, without anginal symptoms for at least 2 months or negative treadmill test at the same time, recruited from consecutive subjects in our outpatient clinics. In addition, we recruited "healthy controls" consisted of hospital staff and relatives, who were defined as "healthy" by careful clinical history and physical examination, ECG, and routine blood tests. The Research Ethics Commitee of our two centres approved the study, and all the subjects gave informed consent to participation.

2.2. Blood Samples and Laboratory Assays. Venepuncture was performed within 48 hours of hospital admission, usually the next morning with the patient fasting for $>12$ hours. In patients with stable CAD and healthy controls, blood samples were taken after overnight fasting and abstinence from tobacco, alcoholic, or caffeine-containing beverages the evening before. Serum fractions were obtained by centrifugation for 15 minutes at $3500 \mathrm{~g}$. Aliquots were stored at $-40^{\circ} \mathrm{C}$ to allow batch analysis in a blinded fashion. Serum levels of hsTnT were assayed by a Cobas 6000 analyser (Roche Diagnostics, Mannheim, Germany). The interassay variation for hsTnT determining was $2.4 \%$, with a lower detection limit of $0.003 \mathrm{ng} / \mathrm{mL}$. We used the established hsTnT cutoff point in ACS in the literature, $0.013 \mathrm{ng} / \mathrm{mL}$ [24]. We also determined copeptin in serum by sandwich immunoluminometric assay (CT-proAVP LIA B.R.A.H.M.S. AG, Hennigsdor, Germany). The detection limit was $0.4 \mathrm{pmol} / \mathrm{L}$ and the interassay variation coefficient $<20 \%$.

2.3. Followup and Adverse Clinical Endpoints. All patients were followed for 12 months by outpatient clinic attendance, telephone contact, and review of the medical notes. We defined "adverse clinical endpoints" as cardiovascular death (death in context of ischaemic or other heart disease, of death with unexplained cause, presumed cardiac), nonfatal 
MI, unstable angina and stroke, and/or admission for acute heart failure.

2.4. Statistical Analysis. Continuous variables were tested for normal distribution by the Kolmogorov-Smirnov test. The normal distributed continuous variables are shown as mean \pm standard deviation, and those nonparametrically distributed are shown as median (interquartile range). Categorical variables are presented as frequencies (percentages). Comparisons of the groups for continuous variables were performed with the unpaired $t$-test for independent samples or the Mann-Whitney $U$ test (as appropriate). The comparison of discrete variables was done by $\chi^{2}$ test or Fisher test (as appropriate). Correlation between two continuous variables was performed using the Spearman test. An ANOVA test (if relevant a Kruskall Wallis test) was performed to assess the differences between biological markers and analysed subject groups. In addition, multiple binary logistic regression analyses were used to determine the clinical and biological factors associated with the presence of elevated biomarkers (levels above their cutoff point). The independent effect of variables on prognosis was calculated using a Cox proportional hazards regression model, incorporating in the multivariate model only those variables that showed $P$ value $<0.15$ in the univariate analysis. The cumulative incidence of adverse clinical events was estimated according to the Kaplan-Meier method, and the log-rank statistic was used for comparisons. A $P<0.05$ was accepted as statistically significant. Statistical analysis was performed using SPSS 15.0 for Windows (SPSS, Inc., Chicago, IL, USA).

\section{Results}

A total of 198 subjects were included for the analyses: 122 patients with non-STEACS and conventional TnT levels $\leq$ $0.035 \mathrm{mg} / \mathrm{mL}, 33$ patients with stable CAD, and 43 healthy controls. The distribution of clinical characteristics and laboratory parameters are listed in Table 1.

3.1. HsTnT. Patients with non-STEACS presented higher serum hsTnT than those with stable CAD and healthy controls [0.010 (0.005-0.023) versus $0.008(0.005-0.011)$; $P=0.036$ and $0.010(0.005-0.023)$ versus $0.004(0.003-$ 0.007); $P<0.001$, resp.)] (Table 1, Figure 1). There was a positive correlation between hsTnT levels and TIMI risk score (Spearman, $r=0.39 P<0.001$ ). There were no differences in hsTnT levels related to current medication on hospital admission (all $P$ values $>0.05$, Table 2 ).

At 12 months of followup, 22 (18.0\%) patients presented adverse clinical events: $7(5.7 \%)$ cardiovascular death, 3 $(2.5 \%)$ nonfatal MI, $9(7.4 \%)$ unstable angina, $2(1.6 \%)$ stroke, and $1(0.8)$ heart failure. We found a significant association between presenting adverse events and showing positive hsTnT levels [OR: 2.99, 95\% IC (1.18-7.57); $P=$ $0.021]$.

There was a significant association between increased hsTnT levels and presenting at least three cardiovascular risk factors, age $>65$ years, ST deviation in the ECG, female sex,

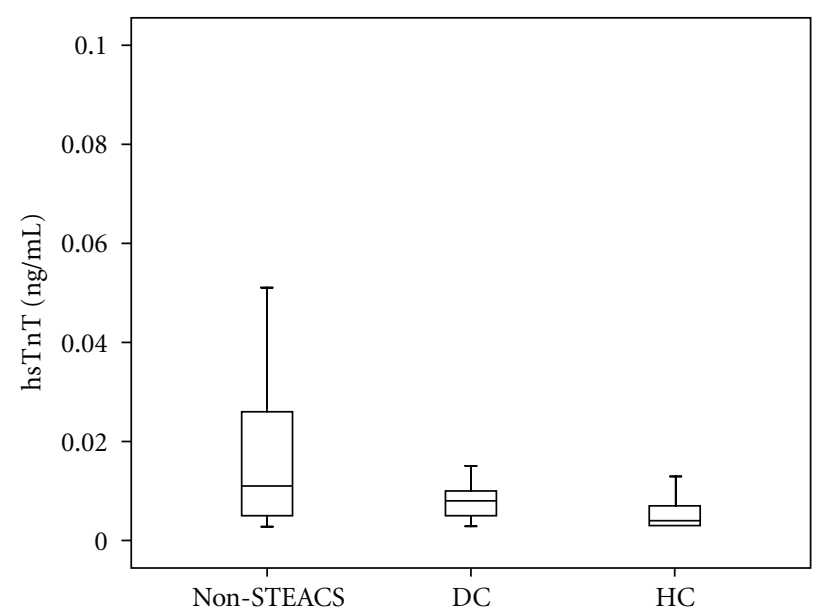

Figure 1: hsTnT levels in non-STEACS, stable coronary disease (disease controls) and healthy controls [values are median (IQR), and non-STEACS: non-ST elevation acute coronary síndrome, DC: disease controls, HC: healthy controls]. $P<0.001$. [Values are median, IQR, and error bars].

and angiographic severe lesions [OR $=4.19$ (95\% CI: $1.76-$ 9.96), $P=0.001 ;$ OR $=2.40$ (95\% CI: $1.21-5.12), P=0.024$; $\mathrm{OR}=5.60$ (95\% CI: $2.44-12.86), P<0.001 ; \mathrm{OR}=0.35(95 \%$ CI: $0.15-0.86, P=0.022$; OR $=5.54$ (95\% CI: $2.07-14.83$ ), $P=0.001$, resp., Table 3]. In the multivariable analysis ST deviation, presenting at least three cardiovascular risk factors, ST deviation, female sex, and severe angiographic lesions remained as independent associated variables with increased hsTnT levels [OR $=3.79$ (95\% CI: 1.02-14.16), $P=0.047 ;$ OR $=6.27$ (95\% CI: $1.98-19.89), P=0.002$; OR $=0.17$ (95\% CI: $0.004-0.68), P=0.013 ;$ OR $=6.41$ (95\% CI: 1.41-29.24), $P=0.016$, resp., Table 3 ).

According to the established cutoff point for hsTnT in ACS $(0.013 \mathrm{ng} / \mathrm{mL})$, we performed univariable Cox regression analysis (Table 4). High serum hsTnT levels [HR 3.29 (95\% CI: $1.33-8.11$ ), $P=0.010$ ], age $>65$ years [HR 3.15 (95\% CI: $1.20-8.31$ ), $P=0.020$ ], and previous non-STEACS [HR 4.35 (95\% CI: 1.68-11.26), $P=0.002$ ] were significant predictors of adverse outcomes. Kaplan Meier curves show that those with hsTnT levels above the cutoff point presented a worse prognosis (log-rank test, $P=0.006$; Figure 2). In a multivariable Cox regression analysis, hsTnT and previous non-STEACS remained as independent predictors of adverse outcomes [HR 2.74 (95\% CI: 1.08-6.95), $P=0.034$; HR 4.02 (95\% CI: $1.55-10.43$ ), $P=0.004$, resp.].

We also explored the prognostic implication of hsTnT in the different scenarios, invasive (64 patients) and conservative approach (58 patients). We found a very relevant prognostic implication for hsTnT levels in patients with the conservative approach [HR 11.45 (95\% CI: 2.93-44.72), $P<0.001$ ], remaining as independent prognostic factor after multivariate analysis (Table 5), whereas this biomarker did not present any relevant implication in the interventional approach in whom coronary catheterism was performed [HR 0.78 (95\% CI: $0.20-3.14), P=0.731$ ]. In the multivariate analysis, only high hsTnT levels and presenting previous 
TABLE 1: Baseline characteristics of patients and controls.

\begin{tabular}{|c|c|c|c|}
\hline & Non-STEACS & Disease controls & Healthy controls \\
\hline$N$ & 122 & 33 & 43 \\
\hline Age $($ mean $\pm \mathrm{SD})$ & $63.2 \pm 11.4$ & $63.9 \pm 12.7$ & $66.62 \pm 7.8$ \\
\hline Male sex (\%) & $83(68.0)$ & $31(93.9)$ & $17(39.5)$ \\
\hline Hypertension (\%) & $92(75.4)$ & $26(78.8)$ & $23(53.5)$ \\
\hline Diabetes mellitus (\%) & $40(32.8)$ & $14(42.4)$ & $10(23.3)$ \\
\hline Hypercholesterolemia (\%) & $67(54.9)$ & $20(60.6)$ & $11(25.6)$ \\
\hline Smoking habit (\%) & $27(22.1)$ & - & - \\
\hline Medications & & - & - \\
\hline Aspirin (\%) & $120(98.4)$ & - & - \\
\hline Clopidogrel (\%) & $120(98.4)$ & - & - \\
\hline ARBs (\%) & $34(27.9)$ & - & - \\
\hline Beta-blocker (\%) & $50(41.0)$ & - & - \\
\hline ACE inhibitors (\%) & $33(27)$ & - & - \\
\hline CA $(\%)$ & $25(20.5)$ & - & - \\
\hline Statins (\%) & $64(52.0)$ & - & - \\
\hline ST deviation (\%) & $39(32)$ & - & - \\
\hline TIMI risk score $($ mean $\pm \mathrm{SD})$ & $2.04 \pm 1.31$ & - & - \\
\hline Catheterism (\%) & $64(52.5)$ & & \\
\hline Stent carrier $(\%)$ & $54(43.9)$ & & \\
\hline hsTnT (ng/mL) & $0.010(0.005-0.023)$ & $0.008(0.005-0.011)$ & $0.004(0.003-0.007)$ \\
\hline Copeptin $>14 \mathrm{pmol} / \mathrm{mL}(\%)$ & $10(8.2)$ & $4(12.1)$ & $1(2.3)$ \\
\hline Copeptin (pmol/L) & $8.42(5.60-13.35)$ & $11.67(7.08-14.56)$ & $6.51(4.80-9.08)$ \\
\hline
\end{tabular}

High-sensitivity troponin T (hsTnT) and copeptin levels data shown as median (IQR). NSTEACS: non ST-elevation acute coronary syndrome; ARBs: angiotensin receptor blockers; ACE: angiotensin-converting enzyme; CA: calcium antagonists.

TABLE 2: hsTnT levels in relation to different modalities of drug therapy.

\begin{tabular}{|c|c|c|}
\hline Therapy & hsTnT levels (ng/mL) & $P$ value \\
\hline \multirow{2}{*}{ Aspirin } & Yes: $0.014(0.007-0.027)$ & \multirow{2}{*}{0.583} \\
\hline & No: 0.008 (0.008-0.008) & \\
\hline \multirow{2}{*}{ Clopidogrel } & Yes: $0.013(0.001-0.026)$ & \multirow{2}{*}{0.383} \\
\hline & No: $0.029(0.029-0.029)$ & \\
\hline \multirow{2}{*}{ ARBs } & Yes: $0.009(0.004-0.016)$ & \multirow{2}{*}{0.557} \\
\hline & No: $0.010(0.005-0.026)$ & \\
\hline \multirow{2}{*}{ Beta-blocker } & Yes: $0.011(0.005-0.026)$ & \multirow{2}{*}{0.478} \\
\hline & No: $0.009(0.004-0.021)$ & \\
\hline \multirow{2}{*}{ ACE inhibitors } & Yes: $0.010(0.005-0.023)$ & \multirow{2}{*}{0.771} \\
\hline & No: $0.009(0.005-0.024)$ & \\
\hline \multirow{2}{*}{ CA } & Yes: $0.012(0.007-0.023)$ & \multirow{2}{*}{0.370} \\
\hline & No: $0.009(0.004-0.021)$ & \\
\hline \multirow{2}{*}{ Statins } & Yes: $0.013(0.005-0.029)$ & \multirow{2}{*}{0.087} \\
\hline & No: $0.009(0.004-0.029)$ & \\
\hline
\end{tabular}

Values are median (IQR). ARBs: angiotensin receptor blockers; ACE: angiotensin-converting enzyme; CA: calcium antagonists.

non-STEACS remained as independent predictors of adverse outcomes at 12-month followup [HR 6.20 (95\% CI: 1.3129.27), $P=0.021$; HR 12.00 (95\% CI: 1.43-100.52), $P=$ 0.022 , resp.].

3.2. Copeptin. Copeptin levels were significantly higher in non-STEACS patients than healthy controls $(P=0.021)$, without differences with disease controls $(P=0.186)$. This biomarker did not show any correlation to classical TnT values obtained at admission (Spearman, $r=0.006, P=$ 0.951). Copeptin levels also were not found to be correlated to TIMI risk score (Spearman, $r=-0.102, P=0.318$ ). We did not find significant association between increased Copeptin levels (above cutoff point of $14 \mathrm{pmol} / \mathrm{L}$ ) and any of 
TABle 3: Association of elevated hsTnT levels with clinical features. Logistic regression analyses. Cutoff point for hsTnT $=0.013 \mathrm{ng} / \mathrm{mL}$.

\begin{tabular}{|c|c|c|c|c|}
\hline \multirow[b]{2}{*}{ Condition } & \multicolumn{2}{|c|}{ Univariate } & \multicolumn{2}{|c|}{ Multivariate } \\
\hline & OR $(95 \% \mathrm{CI})$ & $P$ & OR $(95 \% \mathrm{CI})$ & $P$ \\
\hline age $>65$ years & $2.40(1.21-5.12)$ & 0.024 & $2.42(0.78-7.53)$ & 0.127 \\
\hline Previous non-STEACS & $1.84(0.69-4.86)$ & 0.221 & & \\
\hline Female sex & $0.35(0.15-0.86)$ & 0.022 & $0.17(0.04-0.68)$ & 0.013 \\
\hline$\geq 3 \mathrm{CVRF}$ & $4.19(1.76-9.96)$ & 0.001 & $3.79(1.02-14.16)$ & 0.047 \\
\hline Previous ASA taking & $1.92(0.89-4.15)$ & 0.097 & $0.92(0.26-3.29)$ & 0.893 \\
\hline Severe symptoms & $0.64(0.31-1.55)$ & 0.396 & & \\
\hline ST deviation & $5.60(2.44-12.86)$ & $<0.001$ & $6.27(1.98-19.89)$ & 0.002 \\
\hline PCI at admission & $1.92(0.90-4.10)$ & 0.094 & $2.65(0.75-9.37)$ & 0.131 \\
\hline Angiographic lesions $>50 \%$ & $5.54(2.07-14.83)$ & 0.001 & $6.41(1.41-29.24)$ & 0.016 \\
\hline
\end{tabular}

Previous non-STEACS: Previous non-ST elevation acute coronary syndrome. $\geq 3$ CVRF: at least three cardiovascular risk factors. ASA: acetylsalicylic acid. OR: odds ratio. CI: confidence interval. PCI: percutaneous coronary intervention.

TABLE 4: Cox regression analysis at 12-month followup.

\begin{tabular}{|c|c|c|c|c|}
\hline \multirow[b]{2}{*}{ Condition } & \multicolumn{2}{|c|}{ Univariate } & \multicolumn{2}{|c|}{ Multivariate* } \\
\hline & $\operatorname{HR}(95 \% \mathrm{CI})$ & $P$ & $\operatorname{HR}(95 \% \mathrm{CI})$ & $P$ \\
\hline hsTnT >0.013 ng/mL & $3.29(1.33-8.11)$ & 0.010 & $2.74(1.08-6.95)$ & 0.034 \\
\hline Copeptin > $14 \mathrm{pmol} / \mathrm{L}$ & $0.04(0.00-14.78)$ & 0.285 & & \\
\hline age $>65$ years & $3.15(1.20-8.31)$ & 0.020 & $2.52(0.88-7.21)$ & 0.111 \\
\hline Severe symptoms & $1.65(0.65-4.17)$ & 0.294 & & \\
\hline$\geq 3 \mathrm{CVRF}$ & $2.00(0.75-5.32)$ & 0.167 & & \\
\hline Previous non-STEACS & $4.35(1.68-11.26)$ & 0.002 & $4.02(1.55-10.43)$ & 0.004 \\
\hline ST deviation & $1.14(0.32-3.95)$ & 0.841 & & \\
\hline
\end{tabular}

*Multivariate analysis by conditional method. $\geq 3$ CVRF: at least three cardiovascular risk factors. Previous non-STEACS: previous non-ST elevation acute coronary syndrome. HR: hazard ratio. CI: confidence interval.

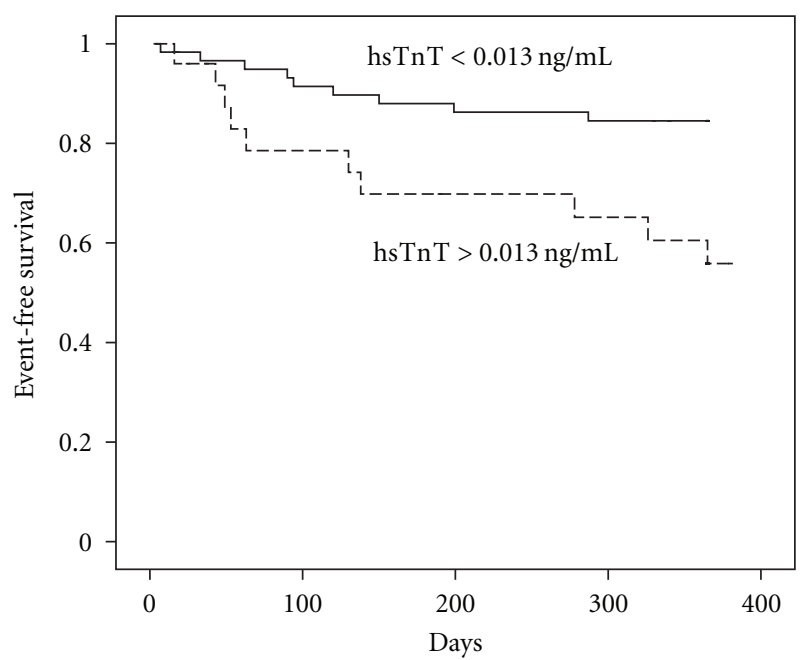

Figure 2: Kaplan-Meier curve showing the effects of hsTnT levels on adverse events following in non-STEACS patients. Patients with raised hs TnT levels, had a significant worse outcome compared with patients with lower hsTnT levels at 12-month followup (cutoff point $>0.0013 \mathrm{ng} / \mathrm{mL}$; log-rank test, $P=0.06$ ).

the studied clinical variables, and Cox analyses did not reveal Copeptin as a significant predictor in non-STEACS patients (Table 4) nor in patients undergoing conservative approach (Table 5).

\section{Discussion}

Although many biomarkers have been investigated in recent years to be used for risk stratification in ACS, cardiac Tn still remains as the preferred and really used biomarker for routine differential cardiovascular diagnosis $[8,9,25]$. Cardiac Tn measured by automated standard assays is superior to all other available biomarkers in clinical practice, including myoglobin, the $\mathrm{MB}$ fraction of creatine kinase, myeloperoxidase, and heart fatty acid-binding protein, for the diagnosis of acute myocardial infarction [26-28]. However, a considerable number of patients cannot still be identified as being at high risk by available routine biomarkers. New high-sensitive cardiac Tn assays have improved precision at the lower limit of detection and have been shown to be very useful in the early diagnosis of acute myocardial infarction [14], but there is a strong debate about the clinical use and limitations of these new Tn high-sensitive detection methods $[22,29,30]$. A recent study by Bonaca et al. showed that low-level increases in Tn I using a sensitive assay identify patients at higher risk of death or MI among patients with non-STEACS, as a probe of the incremental value of newer, more sensitive assays in identifying high-risk patients with ACS [31]. Our hypothesis is that serum hsTnT levels could play an important role for risk stratification, therapeutic decision-making, and prognosis in non-STEACS patients. 
TABLE 5: Cox regression analysis at 12-month followup for patients without invasive catheterization $(n=58)$.

\begin{tabular}{lccc}
\hline & \multicolumn{2}{c}{ Univariate } & Multivariate \\
Condition & HR $(95 \%$ CI $)$ & HR $(95 \%$ CI $)$ & \\
\hline hsTnT $>\mathbf{0 . 0 1 3} \mathbf{~ n g / m L}$ & $11.45(2.93-44.72)$ & $<.20(1.31-29.27)$ & \\
age $>$ 65 years & $1.21(0.35-4.20)$ & 0.760 & 0.416 \\
Copeptin $>14$ pmol/L & $0.039(0.00-95.09)$ & 0.811 & $1.39(0.31-6.20)$ \\
Severe symptoms & $1.18(0.27-4.74)$ & 0.053 & $12.00(1.43-100.52)$ \\
$\geq 3$ CVRF & $3.67(0.98-13.68)$ & $\mathbf{0 . 0 0 7}$ & 0.667 \\
Previous non-STEACS & $17.53(2.19-140.57)$ & 0.202 & $\mathbf{0 . 0 2 2}$ \\
ST deviation & $2.78(0.58-13.44)$ & \\
\hline
\end{tabular}

$\geq 3$ CVRF: at least three cardiovascular risk factors. Previous non-STEACS: previous non-ST elevation acute coronary syndrome. HR: hazard ratio. CI: confidence interval.

The present study shows that patients with non-STEACS and negative conventional $\mathrm{TnT}(>0.035 \mathrm{ng} / \mathrm{mL})$ present a rate of $16 \%$ of adverse events, with a remarkable $4 \%$ rate of death. This highlights that these apparently non- high-risk patients actually require a better risk stratification strategy. Moreover, more than $60 \%$ of our included patients were diagnosed with unstable angina or non-Q acute myocardial infarction, pointing out the moderate risk character of our selected population. These findings are in concordance with those previously described in former studies including similar patients profiles $[32,33]$. In this sense, we show that our non-STEACS patients present significantly higher levels of hsTnT than controls and chronic stable patients. A recent and complete study by Reichlin et al. [14] reveals that hs-Tn assays can substantially improve early diagnostic and allow treatment options for patients presenting with acute myocardial infarction. Now, we add important findings in the utility of hsTnT, as we propose that hsTnT measurement allows the identification and stratification of patients presenting with non-STEACS and provides relevant information about the prognosis at 12-month followup. Hence, we found that patients with raised serum hsTnT levels (above $0.013 \mathrm{ng} / \mathrm{mL}$ ) presented more adverse events than those with lower hsTnT levels. Interestingly, raised hsTnT levels maintained its independent prognostic value, together with the ST deviation and previous aspirin treatment, even after adjusting for other consistent variables. In addition, we also show, for the first time, that raised hsTnT levels are independently associated with cardiovascular risk factors and ST deviation in non-STEACS patients, after adjusting for other confounding variables which is of high relevance since hsTnT could identify patients at higher risk of presenting cardiovascular events.

Nowadays the therapeutic decision in non-STEACS is still basically under the criterium of the cardiologist, although guidelines and recommendations appear in continuous revision of the clinical management of these patients [10, 11]. In the same way, we propose that serum hsTnT levels could help in this treatment decision, since in patients who did not underwent catheterization, those showing high levels of serum hsTnT (cut-off point $0.0225 \mathrm{ng} / \mathrm{mL}$ ) presented a worse outcome. Furthermore, the established cutoff point
$0.0130 \mathrm{ng} / \mathrm{mL}$ did not predict the outcome for these patients. This emphasizes the utility of this biomarker, especially in patients apparently not elective for cardiac catheterization under classical stratification, in which raised hsTnT levels could be indicative for interventionism recommendation.

In order to evaluate the use of biomarkers of different pathophysiologic profiles, we also tested a biomarker of acute endogenous stress, copeptin. Copeptin was found to present predictive value in coronary artery disease [19], and it has been proposed to be of great value in the rapid rule out of acute MI used in combination with $\mathrm{TnT}$ increased levels $[15,19]$. We also study the association between the classical clinical and electrocardiographic parameters in order to achieve a better stratification and managements as well as the prognosis of patients with non-STEACS. We detected significant higher serum copeptin concentration in nonSTEACS' patients in comparison with healthy controls. However, this difference was not significant when comparing with chronic stable patients. In addition, we were not able to find any significant association with clinical parameters or prognostic implications for serum copeptin. One possible reason for the lack of relevant results with copeptin is its rapid release after symptom onset and the normalization of its levels to below the 99th percentile in 10 hours as prevously reported $[16,20]$. Since we collected blood samples within the first $48 \mathrm{~h}$ from the pain onset, it is possible that in most of patients, copeptin detected levels did not correspond with the real raised values within the first fourhour window. Additional studies should be done to clarify copeptin relevance in the first $6 \mathrm{~h}$ from the pain onset in nonSTEACS patients.

Our study has the important limitation of the measurement of single baseline samples in determining prognosis. Although hsTnT levels correlated with TIMI risk score and seems to predict poor outcomes at 12-month followup, we cannot discard changes in hsTnT levels during the time. In addition, the size of our population can also limit the power of our study. Bigger-sized studies are recommended but at this point our data seems to be of great relevance. On the other hand, copeptin levels should be measured within the first four hours form the pain onset in order to reevaluate its clinical relevance in non-STEACS patients. 


\section{Conclusion}

In conclusion, serum hsTnT levels are significantly increased in non-STEACS patients and may be involved in the pathogenesis of this condition. High hsTnT levels improve discrimination of patients at risk of adverse events are predictive of adverse events, and should be included in the therapeutic decision-making.

\section{Abbreviations}

hsTnT: High-sensitivity troponin T

ACS: $\quad$ Acute coronary syndrome

non-STEACS: Non ST-elevation acute coronary syndrome

NT-pro BNP: Amino-terminal pro-B-type natriuretic peptide

CI: $\quad$ Confidence interval

IQR: Interquartile range.

\section{Disclosure}

This study has been partially supported by Roche Diagnostics and B.R.A.H.M.S. Iberia S.L.

\section{Acknowledgments}

This study has been partially supported by a Research Grant from Fundación CajaMurcia and Fundación para la Formación e Investigación Sanitarias de la Región de Murcia. Dr. D. Hernández-Romero holds a postdoctoral position funded by the Instituto de Salud Carlos III. Dr. E. Orenes-Piñero holds a postdoctoral position funded by the Fundación para la Formación e Investigación Sanitarias de la Región de Murcia (FFIS).

\section{References}

[1] R. Ross, "Atherosclerosis-an inflammatory disease," New England Journal of Medicine, vol. 340, no. 2, pp. 115-126, 1999.

[2] D. Tousoulis, G. Davies, C. Stefanadis, P. Toutouzas, and J. A. Ambrose, "Inflammatory and thrombotic mechanisms in coronary atherosclerosis," Heart, vol. 89, no. 9, pp. 993-997, 2003.

[3] E. Falk, "Pathogenesis of Atherosclerosis," Journal of the American College of Cardiology, vol. 47, no. 8, pp. C7-C12, 2006.

[4] E. M. Antman, M. Cohen, P. J. L. M. Bernink et al., "The TIMI risk score for unstable angina/non-ST elevation MI: a method for prognostication and therapeutic decision making," Journal of the American Medical Association, vol. 284, no. 7, pp. 835$842,2000$.

[5] J. A. Castillo Moreno, J. L. Ramos Martín, E. Molina Laborda, S. Egea Beneyto, and J. Ortega Bernal, "Usefulness of clinical profiling and exercise testing in the prognostic assessment of patients admitted with chest pain but without high-risk criteria," Revista Espanola de Cardiologia, vol. 59, no. 1, pp. 1219, 2006.

[6] A. Tello-Montoliu, F. Marín, V. Roldán et al., "A multimarker risk stratification approach to non-ST elevation acute coronary syndrome: Implications of troponin T, CRP, NT pro-BNP and fibrin D-dimer levels," Journal of Internal Medicine, vol. 262, no. 6, pp. 651-658, 2007.

[7] S. Gupta and J. A. de Lemos, "Use and misuse of cardiac troponins in clinical practice," Progress in Cardiovascular Diseases, vol. 50, no. 2, pp. 151-165, 2007.

[8] K. Thygesen and J. S. Alpert, "Universal definition of myocardial infarction," Journal of the American College of Cardiology, vol. 50, no. 22, pp. 2173-2195, 2007.

[9] G. Amodio, G. Antonelli, L. Varraso, V. Ruggieri, and F. Di Serio, "Clinical impact of the troponin 99th percentile cutoff and clinical utility of myoglobin measurement in the early management of chest pain patients admitted to the Emergency Cardiology Department," Coronary Artery Disease, vol. 18, no. 3, pp. 181-186, 2007.

[10] J. P. Bassand, C. W. Hamm, D. Ardissino et al., "Guidelines for the diagnosis and treatment of non-ST-segment elevation acute coronary syndromes: the Task Force for the diagnosis and treatment of non-ST-segment elevation acute coronary syndromes of the European Society of Cardiology," European Heart Journal, vol. 28, no. 13, pp. 1598-1660, 2007.

[11] K. Thygesen, J. Mair, H. Katus et al., "Recommendations for the use of cardiac troponin measurement in acute cardiac care," European Heart Journal, vol. 31, no. 18, pp. 2197-2204, 2010.

[12] S. James, P. Armstrong, R. Califf et al., "Troponin T levels and risk of 30-day outcomes in patients with the acute coronary syndrome: prospective verification in the GUSTO-IV trial," American Journal of Medicine, vol. 115, no. 3, pp. 178-184, 2003.

[13] T. Omland, J. A. De Lemos, M. S. Sabatine et al., "A sensitive cardiac troponin T assay in stable coronary artery disease," New England Journal of Medicine, vol. 361, no. 26, pp. 25382547, 2009.

[14] T. Reichlin, W. Hochholzer, S. Bassetti et al., "Early diagnosis of myocardial infarction with sensitive cardiac troponin assays," New England Journal of Medicine, vol. 361, no. 9, pp. 858-867, 2009.

[15] S. Agewall, E. Giannitsis, T. Jernberg, and H. Katus, "Troponin elevation in coronary vs. non-coronary disease," European Heart Journal, vol. 32, no. 4, pp. 404-411, 2011.

[16] T. Keller, S. Tzikas, T. Zeller et al., "Copeptin improves early diagnosis of acute myocardial infaction," Journal of the American College of Cardiology, vol. 55, no. 19, pp. 2096-2106, 2010.

[17] G. L. Robertson, "Antidiuretic hormone: normal and disordered function," Endocrinology and Metabolism Clinics of North America, vol. 30, no. 3, pp. 671-694, 2001.

[18] J. Struck, N. G. Morgenthaler, and A. Bergmann, "Copeptin, a stable peptide derived from the vasopressin precursor, is elevated in serum of sepsis patients," Peptides, vol. 26, no. 12, pp. 2500-2504, 2005.

[19] S. Q. Khan, O. S. Dhillon, R. J. O'Brien et al., "C-terminal provasopressin (copeptin) as a novel and prognostic marker in acute myocardial infarction: Leicester acute myocardial infarction peptide (LAMP) study," Circulation, vol. 115, no. 16, pp. 2103-2110, 2007.

[20] T. Reichlin, W. Hochholzer, C. Stelzig et al., "Incremental value of copeptin for rapid rule out of acute myocardial infarction," Journal of the American College of Cardiology, vol. 54, no. 1, pp. 60-68, 2009.

[21] F. S. Apple, A. H. B. Wu, and A. S. Jaffe, "European Society of Cardiology and American College of Cardiology guidelines for redefinition of myocardial infarction: How to use existing 
assays clinically and for clinical trials," American Heart Journal, vol. 144, no. 6, pp. 981-986, 2002.

[22] E. Braunwald, E. M. Antman, J. W. Beasley et al., "ACC/AHA 2002 guideline update for the management of patients with unstable angina and non-ST-segment elevation myocardial infarction-summary article: a report of the American College of Cardiology/American Heart Association Task Force on Practice Guidelines (Committee on the Management of Patients with Unstable Angina)," Journal of the American College of Cardiology, vol. 40, no. 7, pp. 1366-1374, 2002.

[23] G. Y. H. Lip and A. D. Blann, "Thrombogenesis, atherogenesis and angiogenesis in vascular disease: a new 'vascular triad", Annals of Medicine, vol. 36, no. 2, pp. 119-125, 2004.

[24] J. L. Januzzi Jr., F. Bamberg, H. Lee et al., "High-sensitivity troponin T concentrations in acute chest pain patients evaluated with cardiac computed tomography," Circulation, vol. 121, no. 10, pp. 1227-1234, 2010.

[25] A. Tello-Montoliu, F. Marín, V. Roldán, and G. Y. H. Lip, “The additive value of biomarkers to clinical risk scores in acute coronary syndrome. Are biomarkers really ready for real world usage?" Heart, vol. 96, no. 3, pp. 227-228, 2010.

[26] D. A. Morrow, E. M. Antman, M. Tanasijevic et al., "Cardiac troponin I for stratification of early outcomes and the efficacy of enoxaparin in unstable angina: a TIMI-11B substudy," Journal of the American College of Cardiology, vol. 36, no. 6, pp. 1812-1817, 2000.

[27] W. Hochholzer, H. J. Buettner, D. Trenk et al., "New definition of myocardial infarction: impact on long-term mortality," American Journal of Medicine, vol. 121, no. 5, pp. 399-405, 2008.

[28] C. J. McCann, B. M. Glover, I. B. A. Menown et al., "Novel biomarkers in early diagnosis of acute myocardial infarction compared with cardiac troponin T," European Heart Journal, vol. 29 , no. 23 , pp. 2843-2850, 2008.

[29] G. Lippi and G. Cervellin, "Letter by lippi and cervellin regarding article, high-sensitivity troponin $\mathrm{T}$ concentrations in acute chest pain patients evaluated with cardiac computed tomography," Circulation, vol. 123, no. 1, article e3, 2011.

[30] J. L. Januzzi Jr., Q. A. Truong, A. A. Mohammed et al., "Reply to letter regarding article, high-sensitivity troponin $t$ concentrations in acute chest pain patients evaluated with cardiac computed tomographyg," Circulation, vol. 123, no. 1, article e4, 2011.

[31] M. Bonaca, B. Scirica, M. Sabatine et al., "Prospective evaluation of the prognostic implications of improved assay performance with a sensitive assay for cardiac troponin I," Journal of the American College of Cardiology, vol. 55, no. 19, pp. 2118-2124, 2010.

[32] O. Danne, M. Möckel, C. Lueders et al., "Prognostic implications of elevated whole blood choline levels in acute coronary syndromes," American Journal of Cardiology, vol. 91, no. 9, pp. 1060-1067, 2003.

[33] N. Gaibazzi, C. Reverberi, and L. Badano, "Usefulness of contrast stress-echocardiography or exercise- electrocardiography to predict long-term acute coronary syndromes in patients presenting with chest pain without electrocardiographic abnormalities or 12-hour troponin elevation," American Journal of Cardiology, vol. 107, no. 2, pp. 161-167, 2011. 


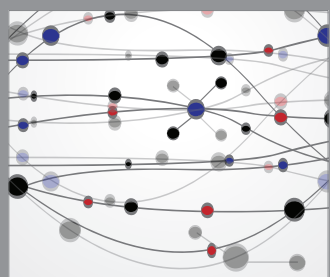

The Scientific World Journal
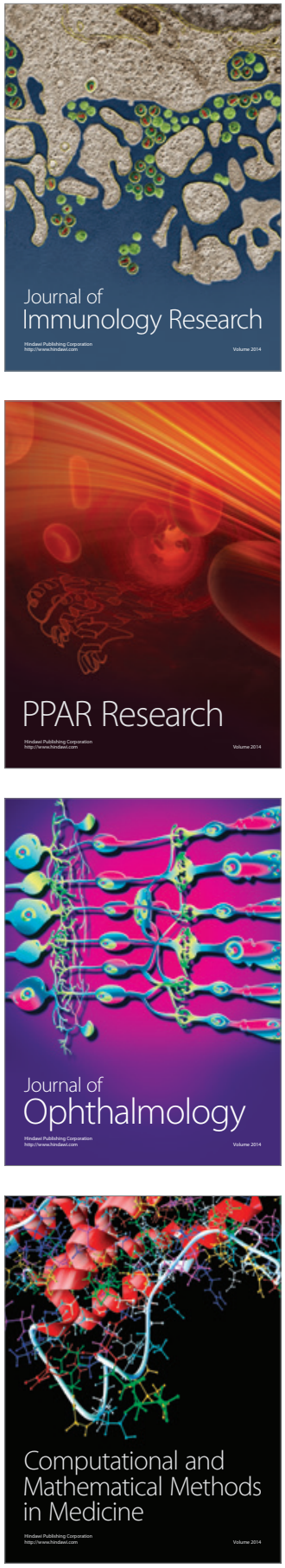



Gastroenterology

Research and Practice
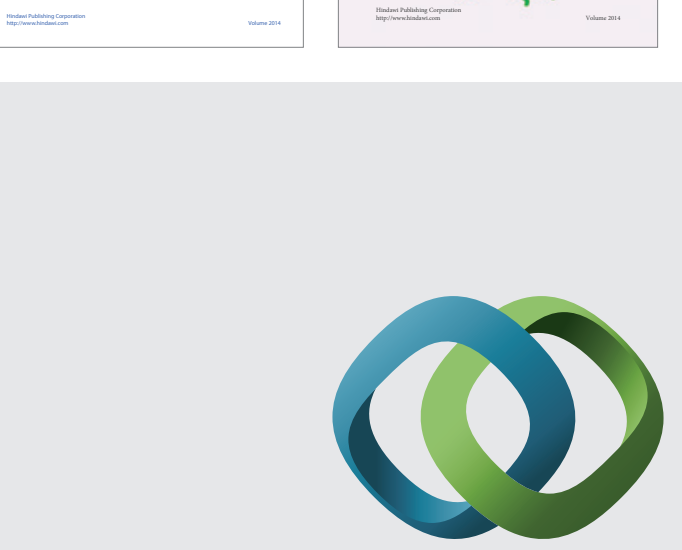

\section{Hindawi}

Submit your manuscripts at

http://www.hindawi.com
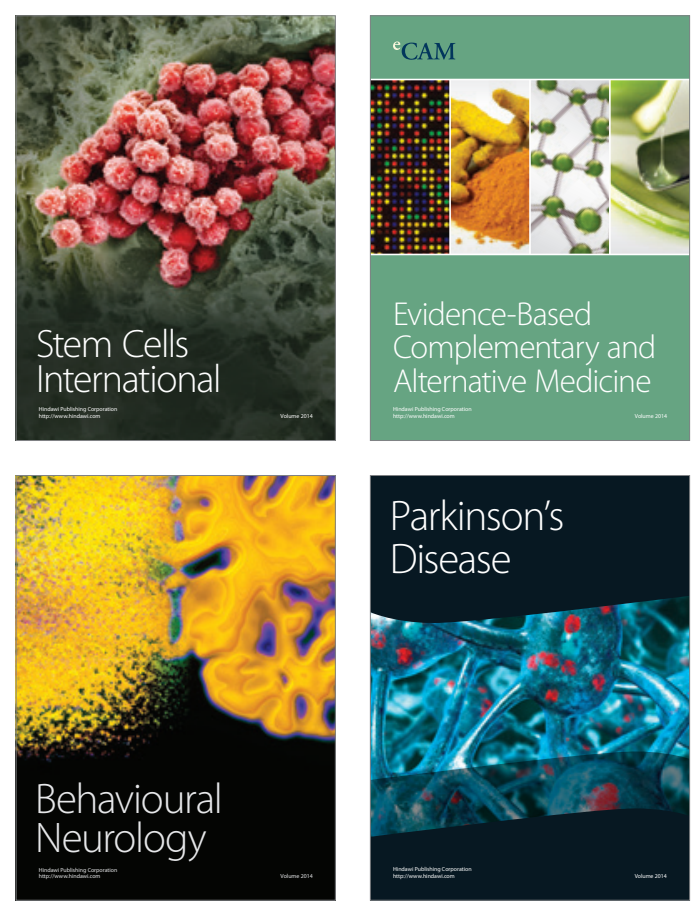

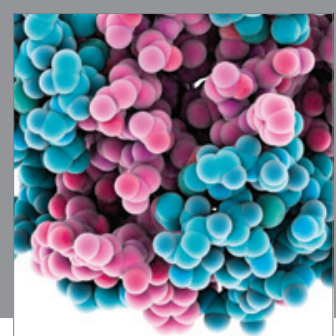

Journal of
Diabetes Research

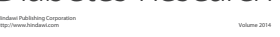



Disease Markers
